\title{
REVIEWS
}

\section{Pancreatic cancer biology and genetics from an evolutionary perspective}

\author{
Alvin Makohon-Moore ${ }^{1,2}$ and Christine A. Iacobuzio-Donahue $e^{2,3,4}$
}

Abstract | Cancer is an evolutionary disease, containing the hallmarks of an asexually reproducing unicellular organism subject to evolutionary paradigms. Pancreatic ductal adenocarcinoma (hereafter referred to as pancreatic cancer) is a particularly robust example of this phenomenon. Genomic features indicate that pancreatic cancer cells are selected for fitness advantages when encountering the geographic and resource-depleted constraints of the microenvironment. Phenotypic adaptations to these pressures help disseminated cells to survive in secondary sites, a major clinical problem for patients with this disease. In this Review we gather the wide-ranging aspects of pancreatic cancer research into a single concept rooted in Darwinian evolution, with the goal of identifying novel insights and opportunities for study.

Driver gene

A gene that confers a selective growth or survival advantage when somatically mutated.
'Sloan Kettering Institute, Memorial Sloan Kettering Cancer Center.

${ }^{2}$ Human Oncology and

Pathogenesis Program,

Memorial Sloan Kettering

Cancer Center.

${ }^{3}$ Department of Pathology,

Memorial Sloan Kettering Cancer Center.

${ }^{4}$ David M. Rubenstein Center for Pancreatic Cancer

Research, Memorial Sloan Kettering Cancer Center, New York, New York 10065, USA.

Correspondence to C.A.I.-D: iacobuzc@mskcc.org

doi:10.1038/nrc. 2016.66

Published online 22 Jul 2016
The question is not what you look at, but what you see. Henry David Thoreau ${ }^{1}$

In the year 2016, an estimated 53,070 patients will be diagnosed with pancreatic ductal adenocarcinoma (hereafter referred to as pancreatic cancer), most of whom will die of their disease within 5 years'. There are no clinically validated screening methods for pancreatic cancer in the curative stage, and surgery remains the only option for cure, despite the fact that only $10-15 \%$ of newly diagnosed patients are deemed eligible ${ }^{3}$. Few other effective treatment modalities exist that significantly extend overall survival ${ }^{4}$. Ultimately, most patients will die with metastases to the liver, lung and/or peritoneum, the most common sites of spread ${ }^{5}$. Patients, clinicians and researchers alike are frustrated at the lack of progress being made, indicating that new strategies are needed to understand this disease.

The term 'cancer' engenders fear and anger, particularly when one is newly faced with the devastating diagnosis of pancreatic cancer. Moreover, a common reaction is to personify the cancer as an evil entity that must be battled to save the patient's life. The weapons for this battle include a surgeon's scalpel, chemotherapy, radiation, targeted agents, holistic approaches and religious faith. But, in a biological sense what really is a pancreatic cancer, or any cancer (BOX 1)? Once the abovementioned preconceived biases are removed, pancreatic cancer reveals itself as a robust example of Darwinian evolution, a pervasive phenomenon in the natural world that is subject to its own rules, restraints and predictable characteristics. Cancer has been discussed in evolutionary terms for 40 years, first by Peter Nowell ${ }^{6}$ in 1976, who proposed clonal evolution as a unifying model of tumour initiation and progression based on his observations in haematopoietic malignancies. However, the importance of evolutionary dynamics for understanding cancer was brought to the forefront by the application of next-generation sequencing methodologies to cancer samples ${ }^{7}$. This has certainly been the case for pancreatic cancer, in which recurrent chromosome abnormalities and subclonal events were described by karyotypic analysis almost two decades ago ${ }^{8}$, whereas the description of intratumoural heterogeneity based on next-generation sequencing was reported only in 2010 (REFS 9, 10).

\section{Three evolutionary stages}

An understanding of pancreatic cancer in evolutionary terms is perhaps best accomplished by characterizing it into three broad stages ${ }^{11}$. These are initiation of the tumour by the acquisition of a driver gene mutation in a cell of origin, clonal expansion of the mutation-carrying cell into a multicellular neoplasm and the introduction of the neoplastic cells into both local and distant microenvironments.

\section{Initiation}

A basic tenet of Darwinian evolution is that purposeless mutations occur randomly in asexually reproducing cells upon which selection then acts ${ }^{12,13}$. For a mutation to occur, a complete cell division must take place. Likewise, the occurrence of a somatic mutation implies that at least one cell division occurred in the lineage that gave rise to that cell ${ }^{12}$. Given that the expected somatic mutation rate is approximately three single nucleotide variants per cell division ${ }^{14}$ and that the adult pancreas is not a 


\section{Box 1 | Evolutionary origins of cancer}

Cancer, defined as the abnormal growth of a clonal population of cells with the ability to invade and destroy surrounding tissues within its host, is a feature of multicellular organisms ${ }^{13}$. This statement is logical, as the development of multicellularity in the Tree of Life required cells to trade off their individual growth potential for the collective good of the population ${ }^{128}$. However, multicellularity alone is not the sole reason why cancer develops. Multicellularity has evolved multiple times throughout the Tree of Life by various mechanisms ${ }^{129}$. In Bacteria, Fungi and Algae, multicellularity has evolved two or more times independently, whereas multicellularity has evolved only once in the Animalia kingdom. Intriguingly, in this instance multicellularity evolved in association with diversification of genes related to integrins, cadherins, WNT signalling, transforming growth factor- $\beta$ (TGF $\beta$ ) signalling and Hedgehog signalling compared with their unicellular ancestors ${ }^{130}$. Thus, the same mechanisms that enabled multicellularity in the Animalia are some of those that, when dysregulated, make the many divergent species, including humans, susceptible to cancerous growth. Neoplastic growths have been documented in the basal metazoan $\mathrm{Hydra}^{131}$, in Drosophila melanogaster ${ }^{132}$ and even in prehistoric species ${ }^{133}$. The extent or frequency with which cancer develops or behaves more aggressively with increasing complexity of multicellular organisms in the Animalia is unknown, and species such as the naked mole rat or elephant have evolved unique mechanisms to resist cancer formation ${ }^{134,135}$. Nonetheless, collectively, cancer may be viewed as a phenomenon of the natural world that represents an evolutionary trade-off of being a multicellular organism descended from metazoans. The fact that most cancers, including pancreatic cancer, occur in humans well past reproductive age supports the idea that the occurrence of cancer has not served as a negative selective force during propagation of our species ${ }^{136}$.

Tree of Life

Standard illustration of branching evolution

encompassing the history of life on Earth in which branch

tips represent extinct or extant species and nodes depict common ancestors.

Familial pancreatic cancer Pancreatic cancer diagnosis in two or more first-degree relatives.

Caretaker genes Genes that when mutated result in a loss of genomic stability and fidelity.

Gatekeeper gene

A gene that when mutated results in a loss of growth control. highly proliferative tissue ${ }^{15}$, by simple chance alone it is exceedingly rare for an initiating driver gene mutation to occur (FIG. 1 a). In patients who develop sporadic pancreatic cancer, the appearance of the driver gene mutation in the first cell is predicted to occur at least two decades before diagnosis?.

Unlike proliferative tissues such as the breast or colon, in which familial cancers occur 10-20 years earlier than sporadic cancers, inheritance of a high-risk variant for developing pancreatic cancer decreases its latency by only 5 years ${ }^{16}$, underscoring that the number of cell divisions and time are important factors in the initiation of pancreatic cancer. Recently, a statistical analysis of numerous cancer types, including pancreatic cancer, determined a strong correlation of lifetime risk with the number of normal stem cell divisions in a tissue $^{17}$. On the basis of estimates of normal pancreatic stem cell renewal rates ${ }^{18,19}$, stochastic mistakes during DNA replication (intrinsic factors) were predicted to substantially contribute to the lifetime risk of pancreatic cancer. However, the relative contribution of intrinsic versus extrinsic factors in cancer initiation has stimulated vigorous scientific debate, with a follow-up study ${ }^{20}$ concluding that the influence of extrinsic factors such as radiation and carcinogens far outweighs that of intrinsic factors. Nonetheless the study by Wu et al. ${ }^{20}$ also showed that nearly half of pancreatic cancer mutations were probably caused by intrinsic factors.

Recent whole-genome sequencing of 593 patients with familial pancreatic cancer indicates that the genetic basis of familial pancreatic cancer is polygenic, that is, many kindreds had one or more high-risk germline variants, but the frequency of any one variant never exceeded $3 \%$ of the population studied ${ }^{21}$. The best-studied germline variants linked to pancreatic cancer risk are $B R C A 1, B R C A 2$, partner and localizer of BRCA2 (PALB2), the Fanconi anaemia genes FANCC and $F A N C G$, and ataxia telangiectasia mutated (ATM), which are all components of the DNA double-strand break repair machinery ${ }^{16,22}$. Mutations in these genes (specifically, BRCA1 and BRCA2) increase genomic instability during faulty homologous recombination at stalled replication forks and hence increase the rate at which somatic mutations occur ${ }^{23}$. Germline mutations in cyclin-dependent kinase (CDK) inhibitor $2 \mathrm{~A}\left(C D K N 2 A\right.$, which encodes $\mathrm{p} 16^{\mathrm{INK} 4 \mathrm{~A}}$ and $\left.\mathrm{p} 19^{\mathrm{ARF}}\right)$, responsible for familial atypical multiple mole melanoma syndrome, are also strongly associated with an increased risk of pancreatic cancer and melanoma ${ }^{16}$, presumably through loss of the G1/S checkpoint. Mutations in DNA double-strand break repair genes probably increase the rate at which the initial driver gene mutation occurs per cell division, consistent with the concept that these are caretaker genes. However, the tumour suppressive function of $\mathrm{p} 16^{\mathrm{INK} 4 \mathrm{~A}}$ indicates that it is a gatekeeper gene ${ }^{24}$. Thus, its loss would be predicted to increase the number of cell divisions, increasing the chance that additional driver gene mutations could occur.

Epidemiological studies point to several risk factors for developing pancreatic cancer that also fit into this framework ${ }^{25}$. For example, patients with chronic pancreatitis owing to protease, serine 1 (PRSS1) or serine peptidase inhibitor, Kazal type 1 (SPINK1) mutations have a well-documented increased risk of developing pancreatic cancer, perhaps as a result of the increased epithelial cell divisions that occur during injury and repair processes, or from reactive oxygen species that cause DNA damage ${ }^{26}$. Both the ongoing cycles of injury and repair and reactive oxygen species would be expected to increase the net number of mutations that occur per division. Inflammatory processes may further enable clonal populations to survive and expand that otherwise would be removed by the immune system ${ }^{27}$. Likewise, smoking contributes mutagens that cause DNA damage in pancreatic cells, thereby promoting the initiating event, clonal expansion and the accumulation of additional mutations as well ${ }^{25,28}$. Obesity is thought to increase pancreatic cancer risk by inducing a chronic pro-inflammatory state and hyperinsulinaemia ${ }^{25,29}$. Type II diabetes, another well-known association with pancreatic cancer, is also thought to increase risk through hyperinsulinaemia and/or the hyperglycaemia caused by the dysregulation of blood glucose levels ${ }^{30}$. Hyperglycaemia may increase cancer risk by supporting the survival and expansion of KRAS mutant clones that have differential dependence on glucose metabolism ${ }^{31}$. Finally, genome-wide association studies have identified various susceptibility loci for pancreatic cancer predicted to modify the net rates of cell growth (telomerase reverse transcriptase (TERT), nuclear receptor subfamily 5 group A member 2 (NR5A2), zinc and ring finger 3 (ZNRF3) and TP63 (Refs 32-34)) or efficiency of DNA repair (structural maintenance of chromosomes $2(\text { SMC2) })^{34}$. 


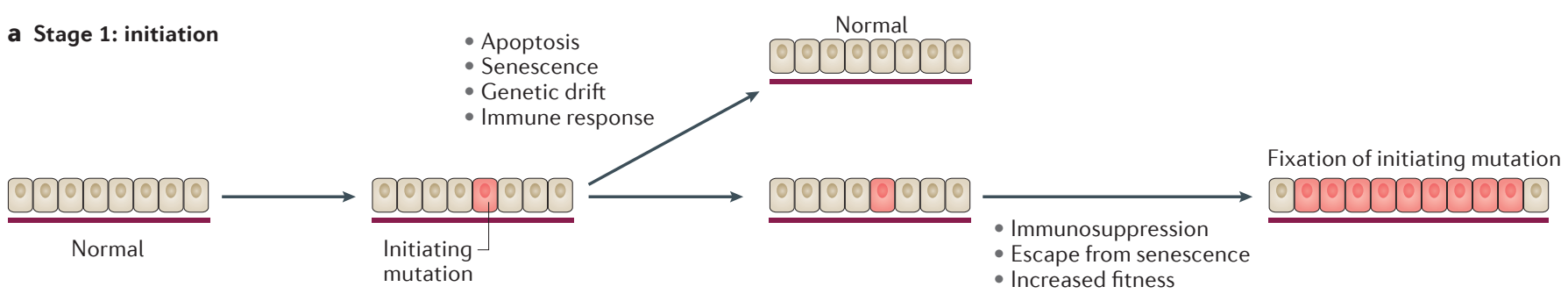

b Stage 2: clonal expansion

Stepwise evolution

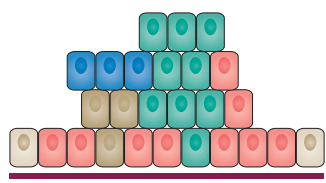

WT

KRAS

$K R A S+C D K N 2 A$

$K R A S+C D K N 2 A+T P 53$

$K R A S+C D K N 2 A+T P 53$ + SMAD4
Punctuated evolution

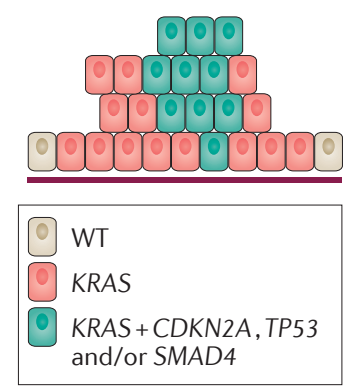

C Stage 3: introduction to foreign microenvironments

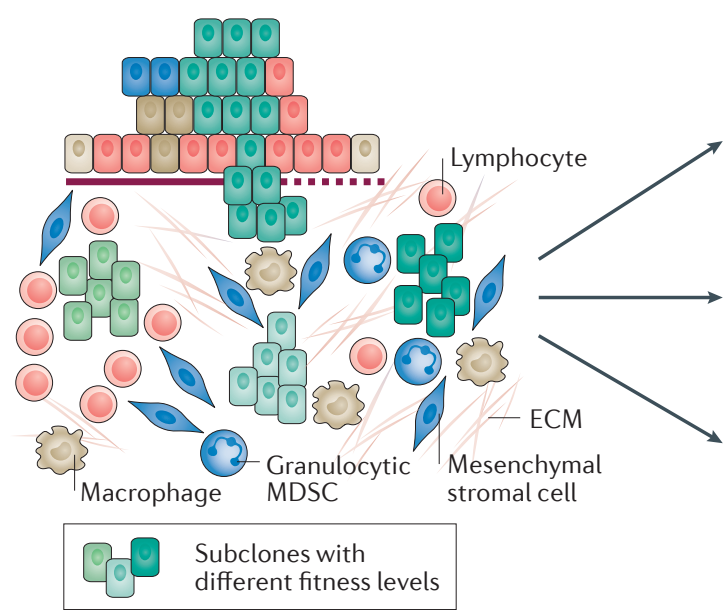

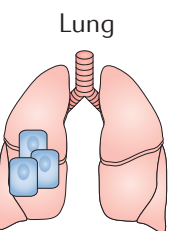

Liver

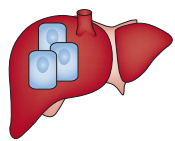

Peritoneum

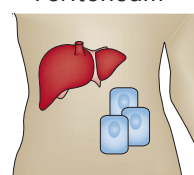

Figure 1 | Stages of pancreatic cancer evolution. a Stage 1: initiation. A normal cell of the pancreas acquires an initiating driver gene mutation as a result of environmental exposure or a lapse in DNA repair. In most instances, this initiating mutation causes the cell to undergo apoptosis or senescence, or to be lost owing to immune surveillance or during a bottleneck event or tissue turnover (genetic drift). If these mechanisms fail, the cell carrying the initiating mutation (red) escapes from senescence and immunosuppression, and continues to fixation because of a survival or growth advantage. $\mathbf{b}$ |Stage 2: clonal expansion. The cell carrying the initiating mutation and its progeny continue to divide, creating a clonal population defined by the presence of the driver gene mutation. In the stepwise progression model, as the population grows in both cell number and geographic space, the descendants gradually acquire additional driver gene mutations (dark beige, blue and green cells in the left panel) and passenger mutations that increase clonal heterogeneity of the neoplasm. In the punctuated evolution model, a catastrophic genome-wide event occurs in a single cell cycle that results in widespread structural damage and acquisition of multiple driver gene alterations simultaneously (green cells in the right panel). c| Stage 3: introduction to foreign microenvironments. Ongoing clonal expansion may lead to a population of cells (green cells) that break through the basement membrane into the surrounding stroma. This event represents a genetic bottleneck that leads to a reduction in genetic diversity. Additional genetic events, signals provided by the stroma, deposition of dense extracellular matrix (ECM) and immune infiltrates all provide selective forces that shape the adaption of these cells into subclonal populations that differ with respect to their overall fitness (represented by cells coloured different shades of green). Dissemination is probably an ongoing process during tumour development; however, the extent to which cells from the entire neoplasm uniformly enter the circulation and/or whether dissemination is restricted to a subpopulation is unknown. Nonetheless, those disseminated cells that achieved high fitness in the primary site may have the greatest chance of colonizing new microenvironments, such as the liver, lung or peritoneum, common sites of metastasis in pancreatic cancer. Colonization of secondary sites represents yet another genetic bottleneck that may further reduce genetic heterogeneity. CDKN2A, cyclin-dependent kinase inhibitor 2A; MDSC, myeloid-derived suppressor cell; WT, wild type.

\section{Clonal expansion}

The occurrence of the initiating driver gene mutation does not guarantee the development of pancreatic cancer, as the mutation must become fixed in the epithelial cell population (FIG. 1a). Up to $33 \%$ of pancreata from autopsy series contain pancreatic intraepithelial neoplasias (PanINs), known precursor lesions of pancreatic cancer (BOX 2), buttressing the notion that most PanINs never progress to an infiltrating carcinoma ${ }^{35}$.
The extent to which the nascent tumour cell then undergoes additional cell divisions enabling the gradual accumulation of somatic alterations over time (stepwise progression, also known as linear progression) or rapidly over a limited number of cell cycles (punctuated) is unknown (FIG. 1b). Support for the stepwise progression model stems from classic evidence demonstrating increasing frequency of KRAS, CDKN2A, TP53 (which encodes p53) and SMAD4 alterations with increasing 
atypia of PanINs ${ }^{36}$. High-sensitivity methods to detect KRAS mutations indicate that they are present in more than $99 \%$ of stage 1 PanIN (PanIN-1) lesions. Moreover, although KRAS mutations are present in all PanIN stages, the proportion of cells containing KRAS mutations increases with PanIN grade, supporting the finding that this population expands clonally ${ }^{37}$. Loss of $\mathrm{p} 16^{\mathrm{INK} 4 \mathrm{~A}}$ protein expression can be demonstrated in PanIN-2 and PanIN-3, with the frequency of $\mathrm{p} 16^{\mathrm{INK} 4 \mathrm{~A}}$ loss higher in PanIN-3 (REF. 38); similarly, TP53 nuclear accumulation or SMAD4 loss has been demonstrated in PanIN-3 and invasive cancers, and the frequency of somatic alteration of both genes is higher in invasive cancer ${ }^{39,40}$. Such patterns indicate waves of clonal expansion in association with the accumulation of driver gene alterations. By contrast, punctuated evolution, or chromothripsis, is defined as the acquisition of numerous structural alterations in a single cell cycle by a catastrophic genomic event ${ }^{41}$. Evidence of chromothripsis has been found in $10 \%$ of pancreatic cancers ${ }^{42}$. Although that study did not characterize the genetic alterations caused by chromothripsis specifically, there was no evidence that chromothripsis was a dominant mechanism of driver gene alteration in those cancers. Thus, although it is entirely plausible that chromothripsis can disrupt driver genes in a stochastic manner, upon which positive selection can act, punctuated evolution does not seem to be as common a pathway as stepwise progression in pancreatic carcinogenesis.

Irrespective of how they accumulate, the genetic landscape of pancreatic cancer is dominated by three or four mountains represented by somatic alterations in KRAS, CDKN2A, TP53 and SMAD4 (REFS 42-45), all of which have been shown to arise in PanINs ${ }^{36,46}$. Advances in sequencing technology and throughput and increasing sample sizes have not altered this terrain, suggesting that the discovery phase of high-frequency genetic targets in pancreatic cancer has reached saturation. It also indicates that there are few evolutionary paths to the formation of pancreatic cancer. However, such efforts continue to be fruitful in identifying previously unknown low-frequency events of significance ${ }^{42,44,45}$. Recurrent somatic alterations are perhaps best understood in the context of the pathway or function affected, for many cases of low-frequency targets reveal themselves to be alternative perturbations of a common pathway ${ }^{43}$ (TABLE 1).

KRAS. Activating mutations of the KRAS oncogene on chromosome $12 \mathrm{p}$ are the most common genetic abnormality, present in approximately $95 \%$ of pancreatic tumours analysed ${ }^{44,47}$. KRAS encodes a member of the

\section{Box 2 | Histological features of pancreatic intraepithelial neoplasia}

Pancreatic intraepithelial neoplasias (PanINs) are pre-invasive neoplasms that arise within the intralobular ducts of the exocrine pancreas. Depending on the extent of the cytological atypia they are classified as PanIN-1 (low-grade dysplasia), PanIN-2 (moderate dysplasia) or PanIN-3 (high-grade dysplasia) (see the figure). A simple cuboidal layer of cells characterizes normal pancreatic ductal epithelium (denoted by arrows in the 'Normal' panel). PanIN-1 can be recognized by mucinous differentiation and elongation of the ductal cells (denoted by the box) despite these cells having minimal nuclear atypia. PanIN-2 lesions have loss of mucinous epithelium in association with nuclear pleomorphism and crowding (arrows). Mitotic figures are more commonly seen at this stage. Finally, PanIN-3 corresponds to frank carcinoma in situ, characterized by pseudopapillary formation (arrow in the bottom panel), a high degree of nuclear atypia, intraluminal apoptotic debris (arrow in the top panel) and frequent mitotic figures. Sequencing of PanlNs for the most common genetic alterations in pancreatic cancer indicates that activating mutations in KRAS are present in more than $99 \%$ of PanlN-1 lesions $^{37}$. Inactivating mutations in cyclin-dependent kinase inhibitor 2A (CDKN2A) can be detected as early as PanIN-2 lesions, and inactivating mutations in TP53 and SMAD4 as early as the PanIN-3 stage ${ }^{36}$. Collectively, these observations have lent bias in the field to the stepwise accumulation of somatic alterations during the clonal expansion phase in some patients (FIG. 1a) but they do not rule out punctuated evolution (FIG. 1 b). Similar progression models have been proposed for two variant precursors of pancreatic cancer, intraductal papillary mucinous neoplasms (IPMNs) and mucinous cystic neoplasms $(\mathrm{MCNs})^{137}$, although the genetic features associated with these lesions are less well characterized. Images courtesy of O. Basturk and G. Askan, Memorial Sloan Kettering Cancer Center, New York, USA. Scale bars, $100 \mathrm{~mm}$.

Normal

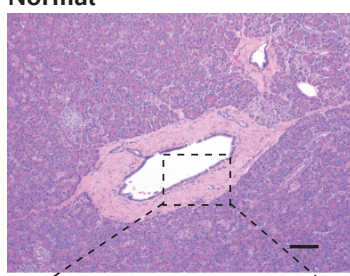

Mitotic figures

Visible, organized

chromosomes in a cell, used as evidence of active mitosis.

Pseudopapillary

Having round outgrowths of tumour cells into the lumen of an epithelial cell-lined duct.
PanIN-1

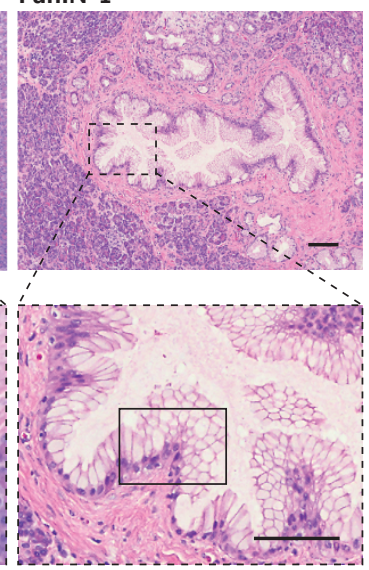

PanIN-2

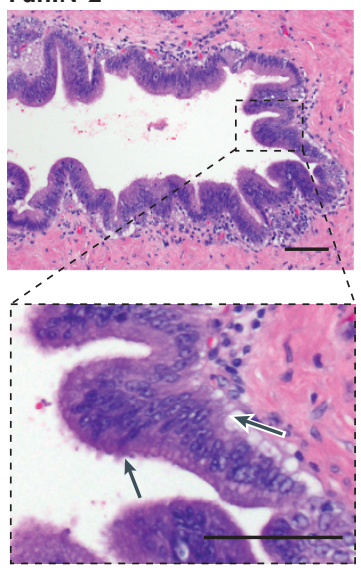

PanIN-3

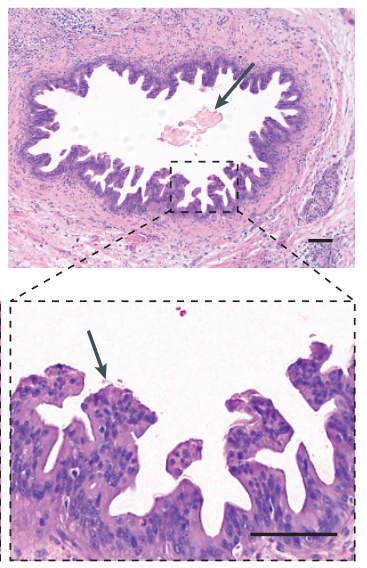


RAS family of GTP-binding proteins that mediate many cellular functions, including proliferation, cell survival and cytoskeletal remodelling ${ }^{48}$. Most mutations in $K R A S$ are believed to cause a constitutively active protein, although recently some KRAS mutants, specifically $K R A S^{\mathrm{G} 12 \mathrm{C}}$, have been demonstrated to have nucleotide cycling activity that is druggable ${ }^{49,50}$. In approximately $4 \%$ of pancreatic cancers KRAS amplification occurs together with the oncogenic mutation ${ }^{42}$. BRAF, the signalling mediator immediately downstream of KRAS, is mutated or amplified in a mutually exclusive manner from $K R A S$ in $3-4 \%$ of cases $^{42,51}$. Intriguingly, although KRAS mutations are found in $99 \%$ of PanIN- $1 \mathrm{~s}^{37}$, no more than $95 \%$ of pancreatic cancers have a KRAS or $B R A F$ mutation, supporting the notion that a KRAS mutation is not strictly required for the development of pancreatic cancer.
CDKN2A. The tumour suppressor gene CDKN2A encodes $\mathrm{p} 16^{\mathrm{INK} 4 \mathrm{~A}}$ and $\mathrm{p} 19^{\mathrm{ARF}}$ through a common locus on chromosome $9 \mathrm{p}^{52}$. The genomic structure of CDKN2A is highly complex in that it produces two mRNAs, each with a unique first exon but sharing exons 2 and 3. However, exon 2 in the mRNA that encodes $\mathrm{p} 19^{\mathrm{ARF}}$ is derived from a different reading frame from that of the mRNA encoding $\mathrm{p} 16^{\mathrm{INK} 4 \mathrm{~A}}$; thus, the two proteins are not isoforms ${ }^{53}$. The high frequency at which this locus is inactivated in pancreatic cancers $(>90 \%)^{54}$ raises the question of which tumour suppressor is being selected for inactivation ${ }^{55}$. Evidence in mice and humans points to $\mathrm{p} 16^{\mathrm{INK} 4 \mathrm{~A}}$ as the primary target because mutations in exon 1 - which is used in the transcript encoding p16 $16^{\mathrm{INK} 4 \mathrm{~A}}-$ that would leave p19 ${ }^{\mathrm{ARF}}$ functional have been reported in both pancreatic cancers and melanomas ${ }^{55}$. However, large homozygous

Table 1 | Major pathways targeted by somatic alterations in the clonal expansion phase of pancreatic cancer*

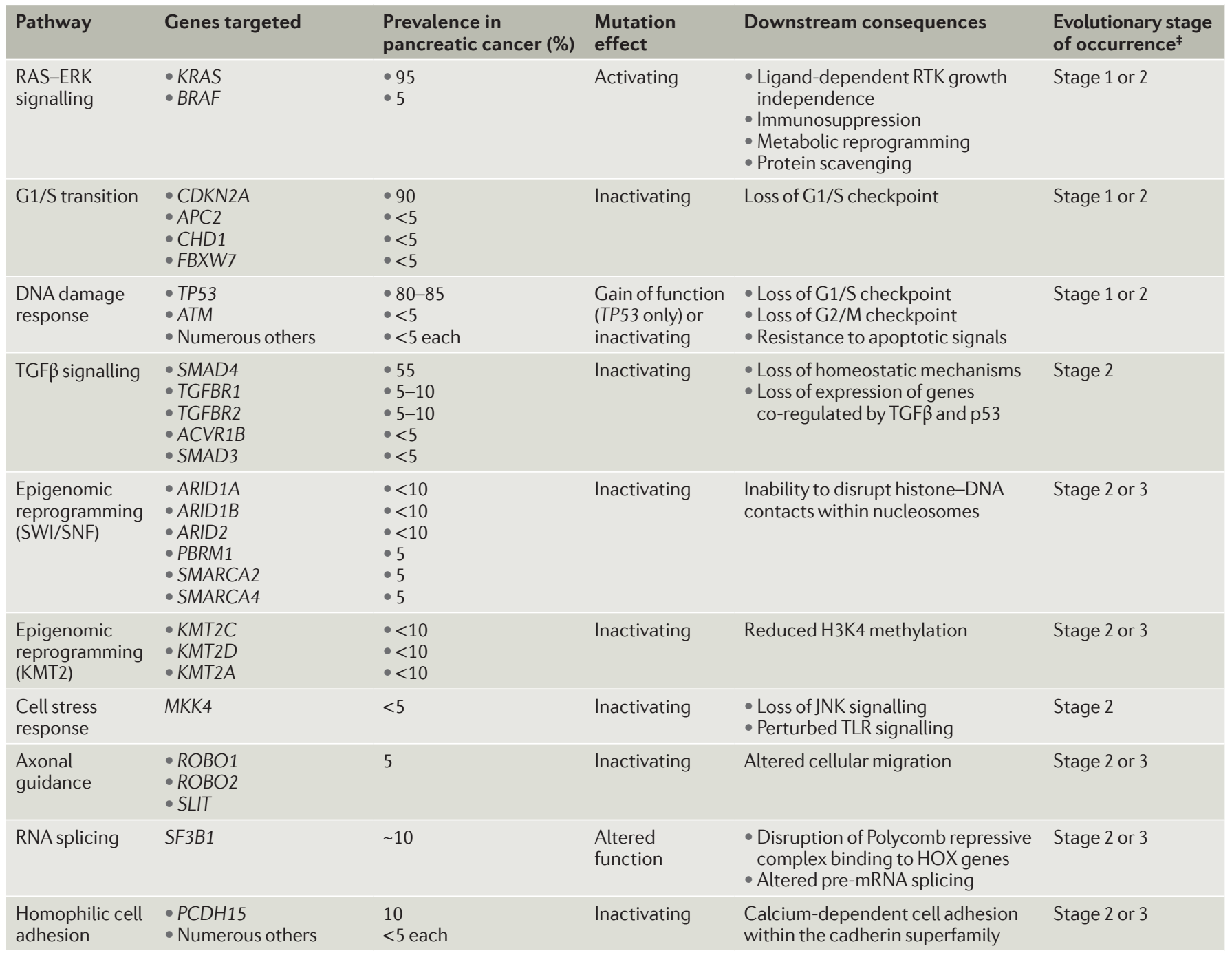

*Data shown are a summary of those described in greater detail in REFS 43,44. ${ }^{\text {*S Stage }} 1$ is the initiation stage, stage 2 is clonal expansion and stage 3 is the introduction to foreign microenvironments. ACVR1B, activin A receptor type $1 \mathrm{~B}$; $A P C 2$, anaphase promoting complex subunit 2; ARID, AT-rich interactive domain-containing; ATM, ataxia telangiectasia mutated; CDKN2A, cyclin-dependent kinase inhibitor 2A; CHD1, chromodomain helicase DNA-binding protein 1; FBXW7, F-box and WD repeat domain containing 7; H3K4, histone H3 lysine 4; HOX, homeobox; JNK, JUN N-terminal kinase; KMT2, histone-lysine $\mathrm{N}$-methyltransferase 2; MKK4, MAPK kinase 4; PBRM1, polybromo 1; PCDH15, protocadherin 15; ROBO, homologue of drosophilia roundabout; SF3B1, splicing factor $3 \mathrm{~b}$ subunit 1; SLIT, homologue of drosophila slit; RTK, receptor tyrosine kinase; SMARCA, SWI/SNF-related matrix-associated actin-dependent regulator of chromatin subfamily A member; TGF $\beta$, transforming growth factor- $\beta$; TGFBR1, TGF $\beta$ receptor 1 ; TLR, Toll-like receptor. 


\section{Fold-back inversions \\ Chromosomal mutations involving a duplication of a genetic sequence followed by an inversion of the copy, resulting in a head-to-head rearrangement.}

\section{Receptor SMADs}

Transcription factors that are activated by extracellular ligands to promote transforming growth factor- $\beta$ (TGF $\beta$ ) signalling and gene expression. deletions often inactivate both proteins so that loss of either may contribute to pancreatic carcinogenesis by different mechanisms. For example, p16 $6^{\mathrm{INK} 4 \mathrm{~A}}$ inhibits cell cycle progression through the G1/S checkpoint mediated by CDKs such as CDK4 and CDK6 (REF. 56). The loss of this important restraint leads to unchecked CDK4 and CDK6 expression and cell cycle progression through the G1/S checkpoint. Telomere shortening in concert with the loss of the G1/S checkpoint creates an environment that facilitates chromosome instability and the accumulation of structural rearrangements, including fold-back inversions, which are a form of mutation relatively specific to pancreatic cancer ${ }^{10,57}$. By contrast, p19 ${ }^{\mathrm{ARF}}$ induces cell-cycle arrest independently of CDKs by binding to the E3 ubiquitin ligase MDM2 to inhibit p53 degradation; loss of p19 ${ }^{\mathrm{ARF}}$ abrogates p53-induced apoptosis and cell cycle arrest ${ }^{55}$. In a small number of pancreatic cancers that retain $C D K N 2 A$, somatic mutations in F-box and WD repeat domain containing 7 (FBXW7), which encodes a ubiquitin ligase that targets cyclin $\mathrm{E}$ for degradation, or the gene encoding the ring E3 ubiquitin ligase anaphase promoting complex subunit 2 ( $A P C 2$, also known as ANAPC2) that regulates chromosome segregation and mitotic fidelity have been reported $^{43,51}$.

TP53. p53 is a latent transcription factor that is activated by stimuli such as DNA damage or stress. Upon activation, p53 performs many functions, including regulation of the G1/S checkpoint, maintenance of G2/M arrest to enable DNA repair, and apoptosis ${ }^{58}$. TP53 is somatically mutated in up to $85 \%$ of pancreatic cancers ${ }^{47}$. As many as $66 \%$ of TP53 mutations in pancreatic cancer are missense mutations that affect the DNA binding domain ${ }^{43,47}$. Although not completely inactivating, such mutations provide oncogenic gains of function compared with the normal protein ${ }^{58}$, often in association with nuclear accumulation of p53 in the neoplastic cell ${ }^{39}$. Previous studies based on immunolabelling for the $\mathrm{p} 53$ protein product in fixed tissues have drastically underestimated the frequency with which TP53 is inactivated by not accounting for somatic alterations that result in a loss of protein expression ${ }^{39,59}$. However, it is clear that nonsense mutations, frameshifts and homozygous deletions not detected by p53 immunolabelling are notable mechanisms of TP53 inactivation in pancreatic cancer ${ }^{43,44}$. In one study of late-stage pancreatic cancers, up to half of all mutations in TP53 were predicted to cause a loss of protein expression leading to null alleles ${ }^{47}$. Among cancers that have no detectable TP53 mutation, numerous genes, such as excision repair cross-complementation group 4 (ERCC4), ERCC6, E1A binding protein p300 (EP300) or RAN binding protein 2 (RANBP2), are mutated at low frequencies and may provide alternative inactivation of one or more $\mathrm{p} 53$ functions. Of particular importance is ATM, a gene also implicated in familial pancreatic cancer that may also be sporadically mutated $^{60}$; ATM phosphorylates p53 directly and has pivotal roles in responding to cell stress and maintaining genome integrity ${ }^{61}$.
SMAD4. SMAD4 is inactivated in approximately $55 \%$ of pancreatic cancers, either by homozygous deletion (30\%) or by an intragenic mutation in association with loss of the second copy $(25 \%)^{62}$. The SMAD4 protein is a crucial co-transcription factor and mediator of the transforming growth factor- $\beta$ (TGF $\beta$ ) canonical signalling pathway for cellular growth, differentiation and maintenance of tissue homeostasis ${ }^{63}$. The TGF $\beta$ pathway is notable for its dualistic nature in cancer; during the early stages of the clonal expansion phase (PanIN-1 and PanIN-2) it restrains neoplastic cell growth, whereas in later stages of clonal expansion (PanIN-3 and invasive cancers) TGF $\beta$ signalling promotes growth, in part owing to the loss of SMAD4 and the canonical arm of the TGF $\beta$ pathway ${ }^{64,65}$. Up to $10 \%$ of pancreatic cancers without $S M A D 4$ alterations harbour an inactivating mutation in TGF $\beta$ receptor 1 (TGFBR1), TGFBR2, activin A receptor type $1 \mathrm{~B}(A C V R 1 B)$ or $S M A D 3$, providing alternative mechanisms to inactivate TGF $\beta$ signalling ${ }^{43,44}$.

An important facet of SMAD4 inactivation is the context in which it occurs. One study that evaluated the patterns of coexistence of driver gene mutations found that most pancreatic cancers with SMAD4 inactivation had coexistent TP53 gain-of-function alterations, whereas pancreatic cancers that retained SMAD4 were more likely to have TP53 loss-of-function alterations ${ }^{47}$. This relationship probably reflects the interdependence of p53 and TGF $\beta$ for transcriptional gene activation (discussed in greater detail in the next section). Thus, TP53 mutant pancreatic cancers can be segregated into two types, those with TP53 loss of function and wildtype SMAD4, and those with TP53 gain of function and $S M A D 4$ loss of function. Validation of these genetic subtypes in independent cohorts and their relationship to therapeutic responses remain to be discerned, including the extent to which these genetic subtypes overlap with other biological subtypes that have been described $^{66-68}$ (BOX 3). Such efforts would be particularly worthwhile in the context of clinical trials of patients with pancreatic cancer for which pretreatment tissues are available ${ }^{69}$.

Synergistic effects between mutations. Although the genes described thus far reveal those pathways that are disrupted, it is unlikely that each gene exerts its effects independently. Mutant KRAS has been shown to impede TGF $\beta$ signalling by inhibiting receptor SMADs ${ }^{70,71}$ and inhibiting $\mathrm{p} 53$ by blocking its amino-terminal phosphorylation $^{72}$. In turn, TGF $\beta$ signalling interacts at many levels with the RAS-RAF-ERK pathway ${ }^{73}$. p53 is required for TGF $\beta$ target gene transactivation by binding to distinct cis-enhancer elements in the same gene promoters through association with receptor SMADs ${ }^{74}$. Furthermore, mutant p53 and SMADs form a complex that inhibits p63, enabling aggressive features of cancer cells ${ }^{75}$. Thus, disruption of crucial driver genes creates a complex tumorigenic network that is expected to greatly alter the systems biology of the cell. An improved understanding of this altered system could be exploited to identify unique vulnerabilities and target specific mutant proteins or pathways ${ }^{76}$. 


\section{Introduction to foreign microenvironments}

Extension of the neoplastic clonal population from the ductal system into the adjacent pancreatic stroma parallels the introduction of a species into a novel environment ${ }^{77,78}$ (FIG. 1c). Invasion into the novel environment is in no way guaranteed however, as the clonal population probably requires a threshold number of genetic, epigenetic and phenotypic alterations to successfully invade and colonize. This concept is exemplified by mouse PanIN-2 and PanIN-3 lesions from which cells may disseminate and survive in the liver but do not persist to form secondary masses ${ }^{79}$. Whether this moment of invasion represents the passive displacement of neoplastic epithelial cells through an incompetent basement membrane, positive selection by one or more microenvironmental factors, or both, is unknown. Regardless, the microenvironment of a primary pancreatic cancer comprises various cell types, extracellular matrix (ECM) components and restricted nutrient and oxygen gradients ${ }^{80-84}$ that act as potent selection forces and shape the ongoing adaptation and continued clonal expansion of this parental clone. In turn, the phenotypes of the neoplastic cells undergo random modifications, one or more of which might support cell survival and maximize fitness in that microenvironment at that moment in time. The result is a primary tumour that is heterogeneous at the cell autonomous and non-cell autonomous levels. Excellent reviews of the pancreatic cancer microenvironment already exist ${ }^{80-84}$; thus, in this section we will focus only on how they relate to evolutionary paradigms. (PSCS). Resident cells of the pancreas that generate fibrous extracellular matrix.
The desmoplastic stroma of pancreatic cancer. The epithelial wound healing response is particularly robust in the pancreas, as evidenced by histology findings in pancreata from patients with chronic pancreatitis ${ }^{85}$. This response is evolutionarily conserved to support the multicellular state and is coordinated in large part by TGF $\beta$ (REF. 86). Features associated with a wound healing response include fibroblast activation, immune suppression, remodelling of the ECM and trophic signals to promote re-epithelialization ${ }^{87}$. That the stroma en masse has an influence on the neoplastic epithelium is undisputed, yet the extent to which each cell type supports rather than restrains neoplastic growth is an area of immense interest.

Co-option of the stromal response by cancer indicates that the stroma provides paracrine signals that select tumour cells with certain properties. Such paracrine signals originate from various sources but have mostly been described for $\alpha$-smooth muscle actin (aSMA) ${ }^{+}$ myofibroblasts. Myofibroblasts are derived from normally quiescent pancreatic stellate cells (PSCs) in the pancreas. Upon activation, PSCs lose their cytoplasmic lipid, transdifferentiate into aSMA ${ }^{+}$myofibroblasts with proliferative capacity, secrete various growth factors, such as TGF $\beta$, fibroblast growth factor (FGF) and plateletderived growth factor (PDGF), and substantially increase production of ECM components ${ }^{88}$. Moreover, unlike the non-neoplastic setting, in which proliferative signals are eventually quelled with the culmination of repair following injury, PSCs and other stromal mesenchymal cells are continuously activated by the neoplastic epithelium itself, which secretes PDGF, TGF $\beta$ and sonic hedgehog $(\mathrm{SHH})^{89-91}$. Evidence supporting the tumour-promoting role of PSCs and their myofibroblastic derivatives comes from mouse studies in which pharmacological inhibition of PSC activation by the vitamin D analogue calcipotriol led to stromal collapse, smaller tumours and improved chemotherapeutic delivery ${ }^{92}$. Analogous results are seen following stromal ablation by short-term inhibition of Hedgehog signalling ${ }^{93}$ or enzymatic ablation of hyaluronic acid (HA), a major constituent of the ECM that is secreted by myofibroblasts ${ }^{94,95}$. By contrast, two recent studies using a mouse model of pancreatic cancer indicated that stromal ablation by conditional deletion of Shh (chronic inhibition) ${ }^{96}$ in or of aSMA ${ }^{+}$myofibroblasts themselves ${ }^{97}$ led to more aggressive tumours. This suggests that distinct components of the myofibroblastic secretome have tumour-restraining properties, although one cannot entirely rule out that the secretomes of other stromal cell populations (for example, macrophages) have tumour suppressive features as well. These opposing forces occur over geographic space and time and in part may underlie the formation of intratumoural heterogeneity by favouring selective sweeps of one clonal population at the expense of another.

The effects of the microenvironment on pancreatic cancer cells go beyond stromal cells. The abundant ECM produced by aSMA ${ }^{+}$myofibroblasts is rich in $\mathrm{HA}$, fibrillar collagens and secreted protein acidic and rich in cysteine (SPARC), which act as a physical barrier to the neoplasm ${ }^{94,95}$. HA is a large negatively 
charged glycosaminoglycan that binds to large amounts of water, leading to high hydrostatic pressure and interstitial fluid pressure (IFP) ${ }^{80}$. The swelling caused by high concentrations of HA stresses collagen fibrils tethered to cancer or endothelial cell surface receptors, which contract in response, leading to pathological IFP, widespread vascular collapse and hypoperfusion. Although this is problematic from the point of view of therapeutic deliver $y^{93,94}$, such a phenomenon itself may cause geographic isolation of neoplastic cells that are already in a nutrient-restricted environment, thus enforcing allopatric evolution and intratumoural heterogeneity. IFP also leads to hypoxia, a pervasive feature of the pancreatic cancer microenvironment that serves as yet another powerful selective force ${ }^{93}$. Pancreatic cancer cells can adapt to these environmental pressures through metabolic reprogramming and shunting of resources; these adaptations occur in association with KRAS mutations well before the onset of invasion and are continuously refined with subclonal evolution ${ }^{83,98}$. This is analogous to ecological studies that have shown that the most successful invasive species are those that are predisposed to the most efficient use of available (limited) resources ${ }^{78}$.

The immune system in pancreatic cancer. The immune system represents yet another highly complex programme that has evolved to support the multicellular state ${ }^{99}$. In the context of cancer evolution immune cells represent native predators. Abundant evidence indicates that the pancreatic cancer microenvironment is immunosuppressed at multiple levels, some of which occur in association with the clonal expansion phase of the neoplasm and themselves may enforce genetic bottlenecks in a temporal and spatial manner ${ }^{100}$. In general, the pancreatic cancer microenvironment is notable for $\mathrm{T}$ cell suppression by several mechanisms, including an accumulation of $\mathrm{CD}^{+}$forkhead box P3 (FOXP3)+ regulatory T cells ( $\mathrm{T}_{\text {reg }}$ cells), M2 tumour-associated macrophages (TAMs), myeloid-derived suppressor cells (MDSCs) and fibroblast activation protein (FAP) + fibroblasts, a type of stromal cell distinct from $\alpha \mathrm{SMA}^{+}$ myofibroblasts in the pancreatic cancer microenvironment ${ }^{84,101,102}$. The endogenous cytotoxic $\mathrm{T}$ cells do not seem to be dysfunctional, as mechanisms to bypass their suppression unmask latent immune responses and promote intratumoural accumulation of T cells ${ }^{101,103}$.

M2 tumour-associated macrophages

(TAMs). Immune cells found in pancreatic tumours that promote inflammation.

Myeloid-derived suppressor cells

(MDSCs). Immune cells of

myeloid origin that regulate the immune response.

Fibroblast activation protein (FAP)+ fibroblasts Stromal cells that commonly react with tumour cells. of metastatic efficiency is determined by the genetic alterations that arise during the clonal expansion phase itself (KRAS, CDKN2A, TP53 and SMAD4), before the moment of invasion ${ }^{47,65}$. Thus, the genetic features of the parental clone play an important part (albeit they are not the only factor) in determining the extent to which the clone will successfully adapt and survive in foreign microenvironments should metastasis occur. Third, in two independent studies encompassing 13 unique patients, metastatic subclones were shown to arise from large populations of cells in the primary tumour ${ }^{10,105}$. These subclones can be identified by their unique set of passenger mutations or structural rearrangements, which are genetic markers of the life history of that lineage ${ }^{9}$. Finally, mathematical models predict at least 5-10 years for the emergence of a metastatic subclone following development of the parental clone, again implying the importance of time for adaptation within the microenvironment ${ }^{9}$. The complement of features of the pancreatic cancer cell or its microenvironment that dictate metastasis to the liver, lungs or peritoneum has yet to be determined. However, recent data using lineage tracing in a mouse model of pancreatic cancer indicate that multiclonal seeding is required to initiate metastases in an organ-specific manner ${ }^{105}$.

\section{Unanswered questions}

Is KRAS the only initiating driver gene in sporadic pancreatic cancer? KRAS is undoubtedly important for pancreatic cancer biology, and extensive efforts are under way to target this oncoprotein ${ }^{106}$. What is debatable is whether KRAS mutations are the only initiating event in pancreatic cancer.

Oncogenic KRAS mutations can be found in the pancreata of patients with no evidence of PanINs or pancreatic cancer, suggesting that they are necessary but not sufficient to initiate pancreatic carcinogenesis $^{107}$. Moreover, people with germline mutations in KRAS have not been reported to have a higher risk of developing pancreatic cancer or other non-neoplastic pancreatic sequelae; instead they develop diseases related to developmental delay, bone marrow failure and syndromic cardio-facio-cutaneous disorders, all probably a result of oncogene-induced senescence ${ }^{108}$. Thus, it seems paradoxical that KRAS mutations that may cause senescence can initiate pancreatic cancer. One explanation for this paradox is that the spectrum of KRAS germline mutations differs with respect to the codons affected and thus they do not lead to KRAS hyperactivity to the same extent as oncogenic mutations, for example, G12S, K117R and A146T mutations, in patients with Costello syndrome compared with G12D mutations in those with pancreatic cancers ${ }^{108}$.

Compelling experimental evidence that supports the notion that mutant KRAS in preductal epithelial cells can initiate pancreatic cancer is its ability to inhibit immune-induced senescence and promote localized immunosuppression ${ }^{27,109,110}$. Oncogenic KRAS has also been shown to induce expression of 
functional interleukin-17 (IL-17) receptors on transformed epithelial cells while stimulating infiltration of IL-17-producing T helper $17\left(\mathrm{~T}_{\mathrm{H}} 17\right)$ cells and $\gamma \delta$ $\mathrm{T}$ cells into the adjacent microenvironment. As a result, the transformed epithelial cells undergo paracrine stimulation by the secreted IL-17, which supports clonal expansion of the KRAS mutant population ${ }^{111}$. Thus, although the random occurrence of an oncogenic KRAS mutation may cause senescence in most instances, occasionally the KRAS mutant cell may survive long enough to incite a local immunotolerant environment that supports its clonal expansion into a large enough population for additional genetic events to occur ${ }^{112}$ (FIG. 1a). This scenario is consistent with mathematical models that predict that at least a decade is required from initiation to formation of the clonal population that will eventually breach the basement membrane and become an infiltrating carcinoma9.

An alternative possibility that should be considered is that mutations in KRAS are not always the initiating event but may be a driver gene alteration that is selected for in the clonal expansion phase following a tumour cell of origin first acquiring a different driver gene mutation. An example of a strong candidate for an alternative initiating driver gene is $C D K N 2 A$, as $C D K N 2 A$ mutations are linked to an inherited risk of pancreatic cancer ${ }^{21}$. Inherited mutations in DNA damage repair genes such as $B R C A 2$ do not negate the possibility that genes such as $C D K N 2 A$ could be an alternative initiating driver, as they act by increasing the number of potentially deleterious genetic events per cell division and hence the chance that inactivating mutations in these driver genes occur. Consistent with this interpretation, there is no difference in the genetics of familial pancreatic cancers compared with those in which the disease occurred sporadically ${ }^{21}$. Finally, although not experimentally studied, constitutional epimutation may be a mechanism of pancreatic carcinogenesis ${ }^{113}$. This would be supported by reports that many patients with a strong familial pattern of inheritance do not have an identifiable germline genetic event ${ }^{21}$. It is crucial to understand these possibilities in light of limited success in screening for pancreatic cancer or in developing chemopreventive strategies thus far. For example, anti-inflammatory or immunomodulatory agents may have a greater preventive effect for KRAS-initiated pancreatic cancers than for those that arise as a result of loss of a tumour suppressive mechanism $^{114}$.

Constitutional epimutation A stably inherited epigenetic alteration that leads to changes in gene expression.

SWI/SNF complex

Evolutionarily ancient group of proteins that remodel

chromatin by altering the positions of nucleosome binding.
Collectively, the frequency of somatic alterations for any member of these gene families is higher, suggesting that a common epigenomic phenotype is selected for by various genotypes. For example, up to $30 \%$ of pancreatic cancers were shown to have an alteration in one of five different members of the SWI/SNF complex in a mutually exclusive manner ${ }^{115}$.

The SWI/SNF family of genes encodes proteins that make up one of two complexes, the BRG1- or HRBM-associated factor (BAF) complex and the polybromo-associated BAF (PBAF) complex. Each complex relies on ATP hydrolysis to directly disrupt histone-DNA contacts. In general the key role for SWI/SNF complexes is to control the balance between differentiation and stemness and to antagonize the action of the Polycomb repressive complex ${ }^{116}$. Although mutations in several SWI/SNF family members have been described in pancreatic cancer, the most frequently mutated genes are AT-rich interactive domain-containing $1 \mathrm{~A}(A R I D 1 A)$ and SWI/SNFrelated matrix-associated actin-dependent regulator of chromatin subfamily A member 2 (SMARCA2), both components of the BAF complex ${ }^{42,44}$. Similar to genes related to SWI/SNF signalling, the KMT2 genes encode proteins that are components of multisubunit complexes. KMT2 proteins methylate histone $\mathrm{H} 3$ on lysine 4 (H3K4) to promote genome accessibility and transcription ${ }^{117}$. In pancreatic cancer, KMT2C (also known as MLL3) is the most commonly mutated member of this family, although mutations in KMT2D (also known as MLL2) and $K M T 2 A$ (also known as $M L L$ ) are also seen ${ }^{42,44}$. Like SWI/SNF, KMT2 genes have pervasive roles in regulating stemness and differentiation.

To date, the temporal occurrence of these gene alterations has not been explored, thus it remains to be seen whether they represent mutations acquired during the clonal expansion phase or subclonal events that are selected for their fitness advantage in the primary tumour microenvironment. This distinction is crucial, as events acquired during early carcinogenesis are expected to be more targetable than those that are subclonal in nature ${ }^{11}$. In addition, unlike CDKN2A, TP53 or SMAD4, in which both alleles are targeted, members of the SWI/SNF and KMT2 gene families require loss of function of only a single allele for their effects in cancer ${ }^{116,117}$. A determination of the requirement of the wild-type allele for cancer cell survival would be fruitful, as it may provide a therapeutic vulnerability using synthetic-lethal approaches ${ }^{118,119}$. The temporal occurrence of these alterations also has importance from the evolutionary perspective. Mutations that arise during the intraductal clonal expansion phase provide a clue to the survival advantage required for the neoplasm to develop, and suggest that at the moment of invasion the parental clone was already maximally equipped for survival through rapid epigenetic adaptation. By contrast, mutations that arise in a subclonal manner after invasion occurs may be a reflection of the spatial heterogeneity of microenvironmental selection factors. Such an instance could 
be exploited to better understand the heterogeneity of the microenvironment in general and in relation to stromal ablation therapies (FIG. 2).

What are the clinically relevant aspects of heterogeneity? Heterogeneity is a loosely used term in cancer biology. At one extreme it may be used to describe inter-patient heterogeneity with respect to biological subtypes of the disease that differ in their aetiology ${ }^{21}$,

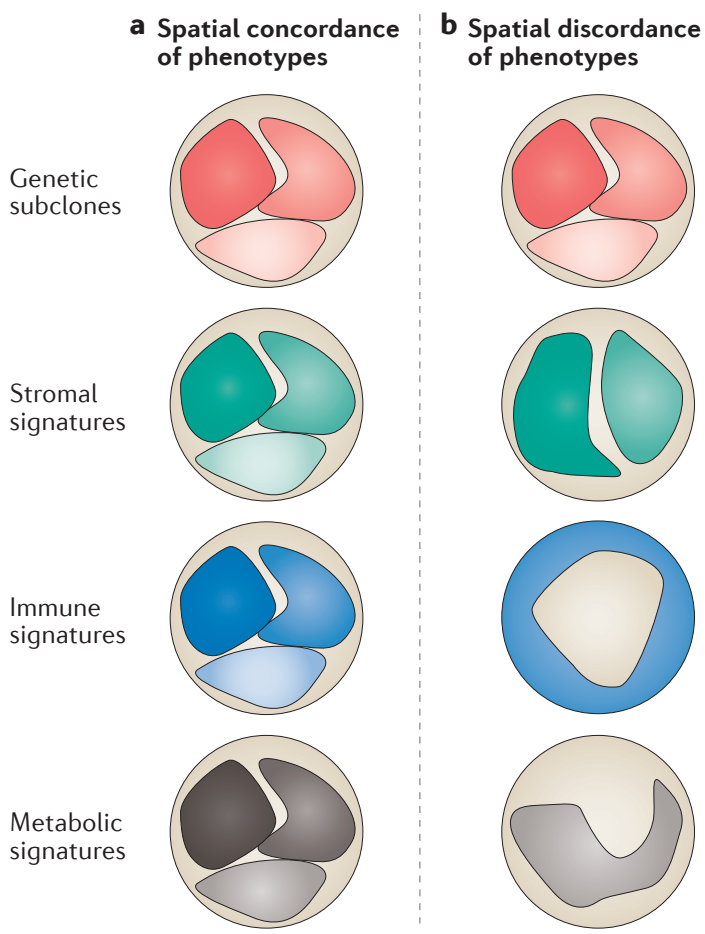

Figure 2 | Geographic heterogeneity in pancreatic cancer. Geographic heterogeneity refers to the spatial variation within a single patient's tumour with respect to genotypes and phenotypes. a I Spatial concordance. In spatial concordance, genetic subclonal populations and regions of distinct stromal biology, immune and/or metabolic phenotypes are geographically linked, as shown in the serial sections of a hypothetical primary tumour. This pattern would be consistent with genotypic heterogeneity driving phenotypic heterogeneity within a neoplasm. A representative example of a genotype that directly drives phenotypic features in pancreatic cancer are KRAS mutations that lead to immunological and metabolic alterations ${ }^{27,142}$. In this scenario, targeting of subclones or the dominant subclone that drives tumour progression may be most efficacious. $\mathbf{b}$ |Spatial discordance. In spatial discordance, genotypic and phenotypic variations are unrelated to each other, implying that phenotypic variations in distinct regions of a tumour are unrelated to genotype and are more influenced by epigenomic or polygenic models of tumour behaviour. Unlike targeting of subclones, in this situation methods to modulate the epigenome to reduce cellular plasticity may have greater value. Spatial discordance has not been formally shown in human tumours because so far all global analyses have relied on single tumour samples, and thus it is of theoretical concern only until proved. biology $y^{66-68}$ or response to therapy ${ }^{42}$ (BOX 3). The success of any personalized intervention depends on the specific genotype and microenvironment, and the immune and metabolic phenotypes of each patient. There is no doubt that a better understanding of such phenotypes will provide rapid improvements in clinical management, as it has already in BRCA-mutant ovarian cancers ${ }^{120}$. At the opposite extreme is intratumoural heterogeneity, most often described in relation to genetics ${ }^{9}$, although epigenetic or phenotypic variants of pancreatic cancer can be described with this term ${ }^{121}$

Broadly in the field of cancer research there is a lack of distinction between genetic subclonal heterogeneity within a primary tumour in general, in metastasisinitiating cells of the primary tumour specifically, or within a metastasis, each of which may have distinct clinical and therapeutic implications at a particular stage of disease ${ }^{122}$ (FIG. 3). A deeper understanding of these different types of heterogeneity will help to define clinically relevant subclones, and the contexts in which subclonal heterogeneity is most meaningful biologically and therapeutically (FIG. 2). Moreover, there is little distinction between heterogeneity of unequivocal driver gene alterations and heterogeneity of somatic alterations with predicted consequences in passenger genes. However, the latter provide unexplored territory with regard to the importance of spatially distinct passenger mutations within a single neoplasm in relation to immunoediting ${ }^{123}$, the mini-driver model of polygenic cancer evolution ${ }^{124}$ or recurrent regions of haploinsufficiency ${ }^{125}$. A counterintuitive view also asks to what extent is heterogeneity reduced during tumour evolution? Although mutations and cell divisions supply heterogeneity over time, there probably exist several bottleneck events that reduce overall diversity during cancer evolution, for example, fixation in evolutionary stage 1 (initiation) and colonization in evolutionary stage 3 (introduction to foreign microenvironments).

What are the evolutionary effects of treatment? Currently, the only potentially curative therapy for pancreatic cancer is surgical removal of the neoplasm ${ }^{3}$, causing an evolutionary effect akin to near total decimation of the cancer cell population. However, most patients who undergo surgery will develop recurrent disease, providing evidence that small populations of cancer cells are left behind either locoregionally or systemically and as predicted by computational models ${ }^{126}$. The evolutionary dynamics by which these residual cells survive, divide and develop into clinically evident populations of cancer cells while under the selective pressures of systemic chemotherapy or radiation is unknown.

The same can be stated for locally advanced, unresectable or metastatic pancreatic cancer. It is reasonable to assume that radiation, cytotoxic chemotherapies and targeted agents that constitute the standards of care for this disease all influence cancer cell evolution ${ }^{13}$. However, at each stage of disease the extent to which different treatment modalities contribute to genetic bottlenecks, 
a Subclonal heterogeneity within a primary tumour
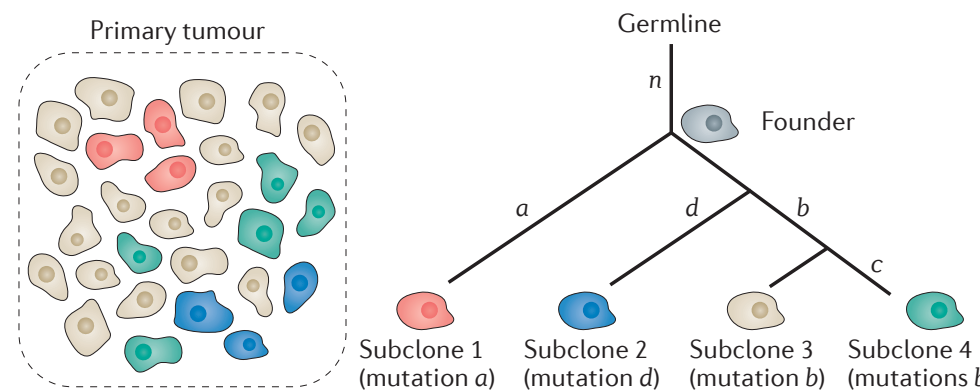

Subclone 1 Subclone $2 \quad$ Subclone 3 Subclone 4 $\begin{array}{llll}(\text { mutation } a) & \text { (mutation } d) & \text { (mutation } b \text { ) } & \text { (mutations } b \text { and } c \text { ) }\end{array}$

b Subclonal heterogeneity within a metastasis
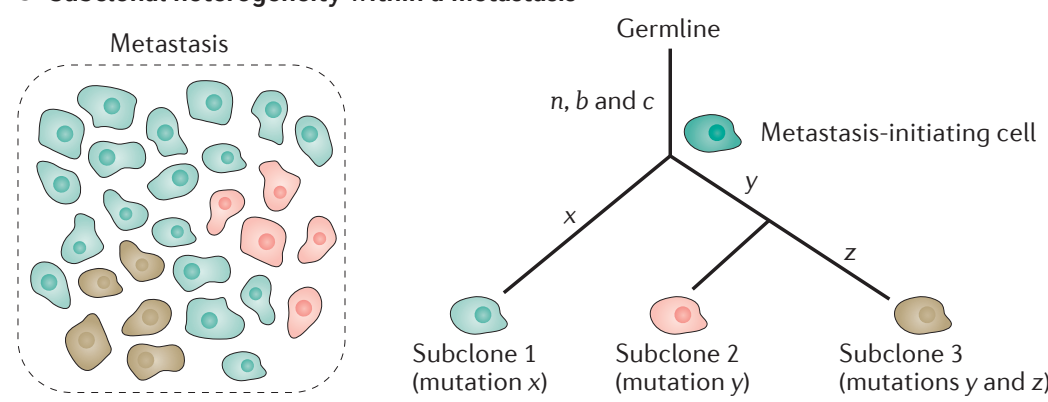

Subclone 1

bclone 2

Subclone 3

(mutations $y$ and $z$ )

C Subclonal heterogeneity of metastasis-initiating cells within a primary tumour

Primary tumour

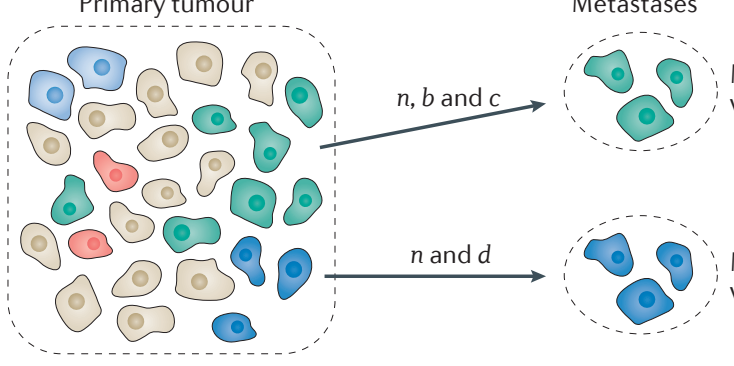

Metastasis-initiating cells with mutation d

Metastasis-initiating cells with mutations $b$ and $c$ the selection of resistant clones or the de novo formation of resistant clones remains unknown despite our general knowledge of resistance mechanisms in cancer ${ }^{127}$. Only with dedicated studies that rely on post-treatment tissues at the time of progression can these questions begin to be addressed.

\section{Summary}

The pace of discovery in understanding pancreatic cancer biology is at its height. Compared with less than one decade ago we have a firm grasp of the genome of pancreatic cancer ${ }^{43,44}$ and the mechanisms by which
4 Figure 3 | The three forms of intratumoural heterogeneity within a patient. a | Intratumoural heterogeneity within a primary tumour. The founder clone (indicated by the grey cell) is the ancestral cell population whose lineage contains all mutations acquired post-fertilization by the most recent common ancestor in the primary tumour. Thus, each mutation that was present in the founder cell is present in every descendant subclone and is inferred by the trunk of the phylogeny that contains $n$ mutations. The founder cell itself no longer exists, as once it divides and accumulates a new mutation or mutations it has evolved. Subclone 1 (red cells) is composed of cells that have acquired mutation $a$. Subclone 2 (blue cells) and subclone 3 (beige cells) are also descendants of the founding cell that have acquired mutations $d$ and $b$, respectively. Subclone 4 (green cells) has mutations $b$ and $c$, indicating that it shares a common ancestor with subclone 3. b | Intratumoural heterogeneity within a metastasis. The metastasis-initiating cell (dark green cell) contains the initial, distinct set of mutations common to all cells of the metastasis (genotype $n$ plus mutations $b$ and $c$ from panel a). The metastasisinitiating cell itself no longer exists, as once it divides and accumulates a new mutation or mutations it has evolved. Subclone 1 (light green cell) represents direct descendants of the metastasis-initiating cell that acquired mutation $x$, and subclone 2 (pink cell) represents the direct descendants that acquired mutation $y$. Subclone 3 (dark beige cell) has mutations $y$ and $z$, indicating that it shares a common ancestor with subclone 2.c | Intratumoural heterogeneity of metastasis-initiating cells within a primary tumour. The metastasis-initiating cells share a common ancestor, yet, nonetheless, have distinct mutations that distinguish one from the other (that is, blue versus green genotypes). As each initiating cell is the ancestral cell for its respective metastasis, every descendant cell will inherit this founding set of mutations.

metabolism is altered to suit the needs of pancreatic cancer cells ${ }^{83}$, and insight into rationally targeting the nodes of immunosuppression ${ }^{102}$ or exploiting genomic instability ${ }^{42}$. However, what is lacking is a convergence of these parallel lines of study, as they are no doubt highly interrelated. This Review has attempted to collate the current understanding of pancreatic cancer into a single concept rooted in evolutionary biology. Mechanisms to support cross-collaboration of these exciting areas of research are expected to further accelerate the pace of discovery and ultimately improve patient survival.
1. Bradford Torrey, E. The Writings of Henry David Thoreau (Riverside Press, 1906).

2. Siegel, R. L., Miller, K. D. \& Jemal, A. Cancer statistics, 2016. CA Cancer J Clin. 66, 7-30 (2016).

3. Winter, J. M. et al. Survival after resection of pancreatic adenocarcinoma: results from a single institution over three decades. Ann. Surg. Oncol. 19 169-175 (2012)

4. Castellanos, E., Berlin, J. \& Cardin, D. B. Current treatment options for pancreatic carcinoma. Curr Oncol. Rep. 13, 195-205 (2011).

5. Yachida, S. \& lacobuzio-Donahue, C. A. The pathology and genetics of metastatic pancreatic cancer. Arch. Pathol. Lab. Med. 133, 413-422 (2009).
6. Nowell, P. C. The clonal evolution of tumor cell populations. Science 194, 23 (1976). Landmark perspective that enumerated the roles of genetic variation, natural selection and evolution in the progression of cancer. Also highlighted how these processes may confound therapeutics.

7. Brosnan, J. A. \& lacobuzio-Donahue, C. A. A new branch on the tree: next-generation sequencing in the study of cancer evolution. Semin. Cell Dev. Biol. 23 , 237-242 (2012).

8. Gorunova, L. et al. Cytogenetic analysis of pancreatic carcinomas: intratumor heterogeneity and nonrandom pattern of chromosome aberrations. Genes Chromosomes Cancer 23, 81-99 (1998).
One of the first studies to catalogue intratumoural karyotypic heterogeneity in pancreatic cancer.

9. Yachida, S. et al. Distant metastasis occurs late during the genetic evolution of pancreatic cancer. Nature 467, 1114-1117 (2010).

Demonstrated that pancreatic cancer metastases evolve from geographic primary tumour subclones. Also modelled the required time for tumour evolution, suggesting that pancreatic cancer takes many years to develop metastases.

10. Campbell, P. J. et al. The patterns and dynamics of genomic instability in metastatic pancreatic cancer Nature 467, 1109-1113 (2010). 
Used phylogenetic approaches to interpret structural rearrangements and the relationship of intrapatient metastases for a set of patients with pancreatic cancer. Also found that genomic instability continues during tumour evolution.

11. Vogelstein, B. \& Kinzler, K. W. The path to cancer three strikes and you're out. N. Engl. J. Med. 373, 1895-1898 (2015).

12. Altrock, P. M., Liu, L. L. \& Michor, F. The mathematics of cancer: integrating quantitative models. Nat. Rev. Cancer 15, 730-745 (2015).

13. Greaves, M. \& Maley, C. C. Clonal evolution in cancer Nature 481, 306-313 (2012)

Modern synthesis of tumour evolutionary concepts and Darwinian selection in cancer.

14. Bozic, l. et al. Accumulation of driver and passenger mutations during tumor progression. Proc. Natl Acad. Sci. USA 107, 18545-18550 (2010)

First to quantify the survival growth advantage conferred by a driver gene mutation.

15. Klein, W. M., Hruban, R. H., Klein-Szanto, A. J. P. \& Wilentz, R. E. Direct correlation between proliferative activity and dysplasia in pancreatic intraepithelial neoplasia (PanIN): additional evidence for a recently proposed model of progression. Mod. Pathol. 15, 441-447 (2002)

16. Petersen, G. M. Familial pancreatic adenocarcinoma Hematol. Oncol. Clin. North Am. 29, 641-653 (2015). Recent and noteworthy review of familial pancreatic cancer genetics, risk assessements and strategies for management.

17. Tomasetti, C. \& Vogelstein, B. Variation in cancer risk among tissues can be explained by the number of stem cell divisions. Science 347, 78-81 (2015).

18. Sangiorgi, E. \& Capecchi, M. R. Bmi 1 lineage tracing identifies a self-renewing pancreatic acinar cell subpopulation capable of maintaining pancreatic organ homeostasis. Proc. Natl Acad. Sci. USA 106 7101-7106 (2009).

19. Furuyama, K. et al. Continuous cell supply from a Sox9-expressing progenitor zone in adult liver, exocrine pancreas and intestine. Nat. Genet. 43 , 34-41 (2011)

20. Wu, S., Powers, S., Zhu, W. \& Hannun, Y. A Substantial contribution of extrinsic risk factors to cancer development. Nature 529, 43-47 (2016).

21. Roberts, N. J. et al. Whole genome sequencing defines the genetic heterogeneity of familial pancreatic cancer Cancer Discov. 6, 166-175 (2016).

Most comprehensive study to date of the germline alterations in a large cohort of patients with familial pancreatic cancer.

22. Bunting, S. F. \& Nussenzweig, A. End-joining, translocations and cancer. Nat. Rev. Cancer 13 0443-454 (2013).

23. Willis, N. A. et al. BRCA1 controls homologous recombination at Tus/Ter-stalled mammalian replication forks. Nature 510, 556-559 (2014).

24. Kinzler, K. W. \& Vogelstein, B. Cancer-susceptibility genes. Gatekeepers and caretakers. Nature 386 761-763 (1997).

\section{Defined how mutations in gatekeeper genes} directly affect tumour growth whereas mutations in caretaker genes create instability that indirectly affects tumour growth.

25. Stolzenberg-Solomon, R. Z. \& Amundadottir, L. T. Epidemiology and inherited predisposition for sporadic pancreatic adenocarcinoma. Hematol. Oncol. Clin. North Am. 29, 619-640 (2015).

26. Weiss, F. U. Pancreatic cancer risk in hereditary pancreatitis. Front. Physiol. 5, 70 (2014).

27. Lee, K. E. \& Bar-Sagi, D. Oncogenic KRas suppresses inflammation-associated senescence of pancreatic ductal cells. Cancer Cell 18, 448-458 (2010). Provided a mechanism by which oncogenic KRAS bypasses senescence to initiate pancreatic cancer.

28. Blackford, A. et al. Genetic mutations associated with cigarette smoking in pancreatic cancer. Cancer Res. 69, 3681-3688 (2009)

29. Gukovsky, I., Li, N., Todoric, J., Gukovskaya, A \& Karin, M. Inflammation, autophagy, and obesity: common features in the pathogenesis of pancreatitis and pancreatic cancer. Gastroenterology 144 1199-1209 (2013).

30. Pannala, R., Basu, A., Petersen, G. M. \& Chari, S. T. Newonset diabetes: a potential clue to the early diagnosis of pancreatic cancer. Lancet Oncol. 10, 288-295 (2009).

31. Micucci, C., Valli, D., Matacchione, G. \& Catalano, A. Current perspectives between metabolic syndrome and cancer. Oncotarget http://dx.doi.org/10.18632/ oncotarget.8341 (2016).
32. Petersen, G. M. et al. A genome-wide association study identifies pancreatic cancer susceptibility loci on chromosomes 13q22.1, 1 q32.1 and 5p15.33. Nat. Genet. 42, 224-228 (2010).

33. Wolpin, B. M. et al. Genome-wide association study identifies multiple susceptibility loci for pancreatic cancer. Nat. Genet. 46, 994-1000 (2014).

34. Childs, E. J. et al. Common variation at 2 p13.3, 3q29, $7 p 13$ and $17 q 25.1$ associated with susceptibility to pancreatic cancer. Nat. Genet. 47, 1911-916 (2015).

35. Kimura, W. How many millimeters do atypical epithelia of the pancreas spread intraductally before beginning to infiltrate? Hepatogastroenterology 50 2218-2224 (2003)

36. Hruban, R. H., Goggins, M., Parsons, J. \& Kern, S. E. Progression model for pancreatic cancer. Clin. Cancer Res. 6, 2969-2972 (2000)

Defined the progression model of pancreatic cancer, beginning with precursor lesions that evolve into invasive carcinoma.

37. Kanda, $\mathrm{M}$. et al. Presence of somatic mutations in most early-stage pancreatic intraepithelial neoplasia. Gastroenterology 142, 2730-733 (2012).

38. Wilentz, R. E. et al. Inactivation of the p16 (INK4A) tumor-suppressor gene in pancreatic duct lesions: loss of intranuclear expression. Cancer Res. 58 4740-4744 (1998)

39. DiGiuseppe, J. A. et al. Overexpression of p53 protein in adenocarcinoma of the pancreas. Am. J. Clin. Pathol. 101, 4684-688 (1994).

40. Wilentz, R. E. et al. Loss of expression of Dpc4 in pancreatic intraepithelial neoplasia: evidence that DPC4 inactivation occurs late in neoplastic progression. Cancer Res. 60, 2002-2006 (2000).

41. Stephens, P. J. et al. Massive genomic rearrangement acquired in a single catastrophic event during cancer development. Cell 144, 2027-40 (2011). Landmark study that defined the genomic features of chromothripsis, a mechanism supporting punctuated evolution in cancer.

42. Waddell, N. et al. Whole genomes redefine the mutational landscape of pancreatic cancer. Nature 518, 495-501 (2015)

43. Jones, S. et al. Core signaling pathways in human pancreatic cancers revealed by global genomic analyses. Science 321, 1801-1806 (2008)

44. Biankin, A. V. et al. Pancreatic cancer genomes reveal aberrations in axon guidance pathway genes. Nature 491, 399-405 (2012).

45. Witkiewicz, A. K. et al. Whole-exome sequencing of pancreatic cancer defines genetic diversity and therapeutic targets. Nat. Commun. 6, 6744 (2015). References 42-45 defined the genomic landscape of pancreatic cancer, the core pathways targeted by somatic alterations and the multiple degrees of structural variation among tumours.

46. Murphy, S. J. et al. Genetic alterations associated with progression from pancreatic intraepithelial neoplasia to invasive pancreatic tumor. Gastroenterology 145 , 1098-1109 (2013).

47. Yachida, S. et al. Clinical significance of the genetic landscape of pancreatic cancer and implications for identification of potential long term survivors. Clin. Cancer Res. 18, 6339-6347 (2012).

48. Pylayeva-Gupta, Y., Grabocka, E. \& Bar-Sagi, D. RAS oncogenes: weaving a tumorigenic web. Nat. Rev. Cancer 11, 1761-774 (2011)

49. Lito, P., Solomon, M., Li, L.-S., Hansen, R. \& Rosen, N. Allele-specific inhibitors inactivate mutant KRAS G12C by a trapping mechanism. Science 351, 604-608 (2016).

50. Patricelli, M. P. et al. Selective inhibition of oncogenic KRAS output with small molecules targeting the inactive state. Cancer Discov. 6, 316-329 (2016).

51. Calhoun, E. S. et al. BRAF and FBXW7 (CDC4, FBW7, AGO, SEL 10) mutations in distinct subsets of pancreatic cancer: potential therapeutic targets. Am. J. Pathol. 163, 1255-1260 (2003)

52. Kim, W. Y. \& Sharpless, N. E. The regulation of INK4/ ARF in cancer and aging. Cell 127, 265-275 (2006).

53. Quelle, D. E., Zindy, F., Ashmun, R. A. \& Sherr, C. J. Alternative reading frames of the INK4a tumor suppressor gene encode two unrelated proteins capable of inducing cell cycle arrest. Cell 83 , 993-1000 (1995).

54. Schutte, M. et al. Abrogation of the Rb/p16 tumorsuppressive pathway in virtually all pancreatic carcinomas. Cancer Res. 57, 3126-3130 (1997).

55. Sharpless, N. E. \& DePinho, R. A. The INK4A/ARF locus and its two gene products. Curr. Opin. Genet. Dev. 9, 22-30 (1999)
56. Bertoli, C. Skotheim, J. M. \& de Bruin, R. A. M. Control of cell cycle transcription during $\mathrm{G} 1$ and $\mathrm{S}$ phases. Nat. Rev. Mol. Cell. Biol. 14, 518-528 (2013).

57. Meeker, A. K. et al. Telomere length abnormalities occur early in the initiation of epithelial carcinogenesis. Clin. Cancer Res. 10, 3317-3326 (2004)

58. Vogelstein, B., Lane, D. $\&$ Levine, A. J. Surfing the p53 network. Nature 408, 307-310 (2000)

59. Hermanova, M. et al. Clinicopathological correlations of cyclooxygenase-2, MDM2, and p53 expressions in surgically resectable pancreatic invasive ductal adenocarcinoma. Pancreas 38, 565-571 (2009).

60. Roberts, N. J. et al. ATM mutations in patients with hereditary pancreatic cancer. Cancer Discov. 2, 41-46 (2012)

61. Herbig, U., Jobling, W. A., Chen, B. P. C., Chen, D. J. \& Sedivy, J. M. Telomere shortening triggers senescence of human cells through a pathway involving ATM, 53, and $\mathrm{p} 21^{\mathrm{CIP} 1}$, but not p16 $6^{\mathrm{INK} 4 \mathrm{a}}$. Mol. Cell 14, 501-513 (2004)

62. Hahn, S. A. et al. DPC4, a candidate tumor suppressor gene at human chromosome 18q21.1. Science 271, 350-353 (1996)

63. Shi, Y. \& Massagué, J. Mechanisms of TGF- $\beta$ signaling from cell membrane to the nucleus. Cell 113 685-700 (2003)

64. Siegel, P. M. \& Massagué, J. Cytostatic and apoptotic actions of TGF $\beta$ in homeostasis and cancer. Nat. Rev. Cancer 3, 807-821 (2003).

65. Whittle, M. C. et al. RUNX3 controls a metastatic switch in pancreatic ductal adenocarcinoma. Cell 161 1345-1360 (2015)

66. Collisson, E. A. et al. Subtypes of pancreatic ductal adenocarcinoma and their differing responses to therapy. Nat. Med. 17, 500-503 (2011).

67. Moffitt, R. A. et al. Virtual microdissection identifies distinct tumor- and stroma-specific subtypes of pancreatic ductal adenocarcinoma. Nat. Genet. 47 1168-1178 (2015)

68. Bailey, P. et al. Genomic analyses identify molecular subtypes of pancreatic cancer. Nature 531, 47-52 (2016).

References 66-68 described phenotypic subtypes of pancreatic cancer based on expression profiling.

69. Crane, C. H. \& lacobuzio-Donahue, C. A. Keys to personalized care in pancreatic oncology. J. Clin. Oncol. 30, 4049-4050 (2012)

70. Saha, D., Datta, P. K. \& Beauchamp, R. D. Oncogenic ras represses transforming growth factor- $\beta / \mathrm{Smad}$ signaling by degrading tumor suppressor Smad4 J. Biol. Chem. 276, 29531-29537 (2001).

71. Kretzschmar, M., Doody, J., Timokhina, I. \& Massagué, J. A mechanism of repression of TGF $\beta$ / Smad signaling by oncogenic Ras. Genes Dev. 13 804-816 (1999).

72. Cordenonsi, M. et al. Integration of TGF- $\beta$ and Ras/ MAPK signaling through p53 phosphorylation Science 315, 843 (2007).

73. Iglesias, M., Frontelo, P., Gamallo, C. \& Quintanilla, M. Blockade of Smad4 in transformed keratinocytes containing a Ras oncogene leads to hyperactivation of the Ras-dependent Erk signalling pathway associated with progression to undifferentiated carcinomas. Oncogene 19, 4134-4145 (2000)

74. Cordenonsi, M. et al. Links between tumor suppressors: p53 is required for TGF- $\beta$ gene responses by cooperating with Smads. Cell 113, 301-314 (2003).

75. Adorno, M. et al. A mutant-p53/Smad complex opposes p63 to empower TGF $\beta$-induced metastasis. Cell 137, 87-98 (2009).

76. Rubinson, D. A. \& Wolpin, B. M. Therapeutic approaches for metastatic pancreatic adenocarcinoma. Hematol. Oncol. Clin. North Am. 29 761-776 (2015)

77. Richardson, D. M. \& Pyšek, P. Naturalization of introduced plants: ecological drivers of biogeographical patterns. New Phytol. 196, 383-396 (2012)

78. Suarez, A. V. \& Tsutsui, N. D. The evolutionary consequences of biological invasions. Mol. Ecol 17 351-360 (2008)

79. Rhim, A. D. et al. EMT and dissemination precede pancreatic tumor formation. Cell 148, 349-361 (2012)

80. Stromnes, I. M., DelGiorno, K. E., Greenberg, P. D. \& Hingorani, S. R. Stromal reengineering to treat pancreas cancer. Carcinogenesis 35, 1451-1460 (2014).

81. Neesse, A., Algül, H., Tuveson, D. A. \& Gress, T. M. Stromal biology and therapy in pancreatic cancer: a changing paradigm. Gut 64, 1476-1484 (2015). 
82. Cohen, R. et al. Targeting cancer cell metabolism in pancreatic adenocarcinoma. Oncotarget 6 , 16832-16847 (2015)

83. Sousa, C. M. \& Kimmelman, A. C. The complex landscape of pancreatic cancer metabolism. Carcinogenesis 35, 1441-1450 (2014).

84. Vonderheide, R. H. \& Bayne, L. J. Inflammatory networks and immune surveillance of pancreatic carcinoma. Curr. Opin. Immunol. 25, 200-205 (2013).

85. Ceyhan, G. O. \& Friess, H. Pancreatic disease in 2014 pancreatic fibrosis and standard diagnostics. Nat. Rev. Gastroenterol. Hepatol 12, 68-70 (2015).

86. Gurtner, G. C., Werner, S., Barrandon, Y. \& Longaker, M. T. Wound repair and regeneration. Nature 453, 314-321 (2008).

87. Dvorak, H. F. Tumors: wounds that do not heal. Similarities between tumor stroma generation and wound healing. N. Engl. J. Med. 315, 1650-1659 (1986).

88. Masamune, A. \& Shimosegawa, T. Pancreatic stellate cells: a dynamic player of the intercellular communication in pancreatic cancer. Clin. Res. Hepatol Gastroenterol. 39 (Suppl. 1), S98-S103 (2015).

89. Bailey, J. M. et al. Sonic hedgehog promotes desmoplasia in pancreatic cancer. Clin. Cancer Res. 14, 5995-6004 (2008).

90. Bachem, M. G. et al. Pancreatic carcinoma cells induce fibrosis by stimulating proliferation and matrix synthesis of stellate cells. Gastroenterology 128 907-921 (2005)

91. Taeger, J. et al. Targeting FGFR/PDGFR/VEGFR impairs tumor growth, angiogenesis, and metastasis by effects on tumor cells, endothelial cells, and pericytes in pancreatic cancer. Mol. Cancer Ther. 10, 2157-2167 (2011).

92. Sherman, M. H. et al. Vitamin D receptor-mediated stromal reprogramming suppresses pancreatitis and enhances pancreatic cancer therapy. Cell 159, 80-93 (2014).

93. Olive, K. P. et al. Inhibition of Hedgehog signaling enhances delivery of chemotherapy in a mouse model of pancreatic cancer. Science 324, 1457-1461 (2009).

Demonstrated that poor vascularization and perfusion may contribute to inefficient therapy delivery in pancreatic tumours, leading to primary resistance.

94. Provenzano, P. P. et al. Enzymatic targeting of the stroma ablates physical barriers to treatment of pancreatic ductal adenocarcinoma. Cancer Cell 21, 418-429 (2012)

95. Jacobetz, M. A. et al. Hyaluronan impairs vascular function and drug delivery in a mouse model of pancreatic cancer. Gut 62, 112-120 (2013).

96. Rhim, A. D. et al. Stromal elements act to restrain, rather than support, pancreatic ductal adenocarcinoma. Cancer Cell 25, 735-747 (2014).

97. Özdemir, B. C. et al. Depletion of carcinomaassociated fibroblasts and fibrosis induces immunosuppression and accelerates pancreas cancer with reduced survival. Cancer Cell 25, 719-734 (2014).

98. White, E. Exploiting the bad eating habits of Rasdriven cancers. Genes Dev. 27, 2065-2071 (2013).

99. Flajnik, M. F. \& Kasahara, M. Origin and evolution of the adaptive immune system: genetic events and selective pressures. Nat. Rev. Genet. 11, 47-59 (2010).

100. Clark, C. E. et al. Dynamics of the immune reaction to pancreatic cancer from inception to invasion. Cancer Res. 67, 9518-9527 (2007).

Characterized the immune response during tumou evolution using a mouse model of pancreatic cancer.

101. Feig, C. et al. Targeting CXCL12 from FAP-expressing carcinoma-associated fibroblasts synergizes with
anti-PD-L1 immunotherapy in pancreatic cancer. Proc. Natl Acad. Sci. USA 110, 20212-20217 (2013)

102. Beatty, G. L. et al. CD40 agonists alter tumor stroma and show efficacy against pancreatic carcinoma in mice and humans. Science 331, 1612-1616 (2011)

103. Foley, K., Kim, V., Jaffee, E. \& Zheng, L. Current progress in immunotherapy for pancreatic cancer. Cancer Lett. http://dx.doi.org/10.1016/j. canlet.2015.12.020 (2015).

104. Fidler, I. J. Metastasis: quantitative analysis of distribution and fate of tumor emboli labeled with 125 I-5-iodo-2'-deoxyuridine. J. Natl Cancer Inst. 45, 773-782 (1970).

105. Maddipati, R. \& Stanger, B. Z. Pancreatic cance metastases harbor evidence of polyclonality. Cancer Discov. 5, 1086-1097 (2015).

106. Cox, A. D., Fesik, S. W., Kimmelman, A. C., Luo, J. \& Der, C. J. Drugging the undruggable RAS: mission possible? Nat. Rev. Drug Discov. 13, 828-851 (2014).

107. Maire, F. et al. Differential diagnosis between chronic pancreatitis and pancreatic cancer: value of the detection of KRAS2 mutations in circulating DNA. Br. J. Cancer 87, 551-554 (2002)

108. Schubbert, S., Shannon, K. \& Bollag, G. Hyperactive Ras in developmental disorders and cancer. Nat. Rev. Cancer 7, 295-308 (2007)

109. Pylayeva-Gupta, Y., Lee, K. E., Hajdu, C. H., Miller, G $\&$ Bar-Sagi, D. Oncogenic Kras-induced GM-CSF production promotes the development of pancreatic neoplasia. Cancer Cell 21, 836-847 (2012).

110. Zhang, Y. et al. CD4 ${ }^{+}$T lymphocyte ablation prevents pancreatic carcinogenesis in mice. Cancer Immunol. Res. 2, 423-435 (2014)

111. McAllister, F. et al. Oncogenic Kras activates a hematopoietic-to-epithelial IL-17 signaling axis in preinvasive pancreatic neoplasia. Cancer Cell 25, 621-637 (2014).

112. Morton, J. P. et al. Mutant p53 drives metastasis and overcomes growth arrest/senescence in pancreatic cancer. Proc. Natl Acad. Sci. USA 107, 246-251 (2010).

113. Hitchins, M. P. Constitutional epimutation as a mechanism for cancer causality and heritability? Nat. Rev. Cancer 15, 625-634 (2015)

114. Cui, X.-J. et al. High-dose aspirin consumption contributes to decreased risk for pancreatic cancer in a systematic review and meta-analysis. Pancreas 43 , 135-140 (2014).

115. Shain, A. H. et al. Convergent structural alterations define SWItch/Sucrose NonFermentable (SWI/SNF) chromatin remodeler as a central tumor suppressive complex in pancreatic cancer. Proc. Natl Acad. Sci. USA 109, E252-E259 (2012).

116. Masliah-Planchon, J., Bièche, I., Guinebretière, J.-M. Bourdeaut, F. \& Delattre, O. SWI/SNF chromatin remodeling and human malignancies. Annu. Rev. Pathol. 10, 145-171 (2015).

117. Rao, R. C. \& Dou, Y. Hijacked in cancer: the KMT2 (MLL) family of methyltransferases. Nat. Rev. Cancer 15, 334-346 (2015).

118. Bitler, B. G. et al. Synthetic lethality by targeting EZH2 methyltransferase activity in ARID1 A-mutated cancers. Nat. Med. 21, 231-238 (2015).

119. Helming, K. C. et al. ARID1B is a specific vulnerability in ARID1 A-mutant cancers. Nat. Med. 20, 251-254 (2014).

120. Kaufman, B. et al. Olaparib monotherapy in patients with advanced cancer and a germline BRCA $1 / 2$ mutation. J. Clin. Oncol. 33, 244-250 (2015).

121. Robertson-Tessi, M., Gillies, R. J., Gatenby, R. A. \& Anderson, A. R. A. Impact of metabolic heterogeneity on tumor growth, invasion, and treatment outcomes. Cancer Res. 75, 1567-1579 (2015).

122. Vogelstein, B. et al. Cancer genome landscapes. Science 339, 1546-1558 (2013).
123. Snyder, A., Wolchok, J. D. \& Chan, T. A. Genetic basis for clinical response to CTLA-4 blockade. N. Engl. J. Med. 372, 783 (2015).

124. Castro-Giner, F., Ratcliffe, P. \& Tomlinson, I. The minidriver model of polygenic cancer evolution. Nat. Rev. Cancer 15, 680-685 (2015)

125. Wang, L. et al. Whole-exome sequencing of human pancreatic cancers and characterization of genomic instability caused by MLH 1 haploinsufficiency and complete deficiency. Genome Res. 22, 208-219 (2012).

126. Haeno, H. et al. Computational modeling of pancreatic cancer reveals kinetics of metastasis suggesting optimum treatment strategies. Cell 148, 362-375 (2012)

127. Gillies, R. J., Verduzco, D. \& Gatenby, R. A Evolutionary dynamics of carcinogenesis and why targeted therapy does not work. Nat. Rev. Cancer 12 487-493 (2012)

128. Niklas, K. J. The evolutionary-developmental origins of multicellularity. Am. J. Bot. 101, 6-25 (2014).

129. Niklas, K. J. \& Newman, S. A. The origins of multicellular organisms. Evol. Dev. 15, 41-52 (2013)

130. Rokas, A. The origins of multicellularity and the early history of the genetic toolkit for animal development. Annu. Rev. Genet. 42, 235-251 (2008).

131. Domazet-Lošo, T. et al. Naturally occurring tumours in the basal metazoan Hydra. Nat. Commun. 5, 4222 (2014).

132. Gateff, E. Malignant neoplasms of genetic origin in Drosophila melanogaster. Science 200, 1448-1459 (1978).

133. Natarajan, L. C., Melott, A. L., Rothschild, B. M. \& Martin, L. D. Bone cancer rates in dinosaurs compared with modern vertebrates. Trans. Kans. Acad. Sci. 110, 155-158 (2007)

134. Tian, X. et al. High-molecular-mass hyaluronan mediates the cancer resistance of the naked mole rat. Nature 499, 346-349 (2013)

135. Abegglen, L. M. et al. Potential mechanisms for cancer resistance in elephants and comparative cellular response to DNA damage in humans. JAMA 314 1850-1860 (2015).

136. Vasseur, E. \& Quintana-Murci, L. The impact of natural selection on health and disease: uses of the population genetics approach in humans. Evol. Appl. 6, 596-607 (2013).

137. Matthaei, H., Schulick, R. D., Hruban, R. H. \& Maitra, A. Cystic precursors to invasive pancreatic cancer. Nat. Rev. Gastroenterol. Hepatol 8, 141-150 (2011).

138. Chantrill, L. A. et al. Precision medicine for advanced pancreas cancer: the individualized molecula pancreatic cancer therapy (IMPaCT) trial. Clin. Cancer Res. 21, 2029-2037 (2015).

139. Hoadley, K. A. et al. Multiplatform analysis of 12 cancer types reveals molecular classification within and across tissues of origin. Cell 158, 929-944 (2014).

140. Rech, A. J. et al. CD25 blockade depletes and selectively reprograms regulatory $T$ cells in concert with immunotherapy in cancer patients. Sci. Transl Med. 4, 134ra62 (2012).

141. Soares, K. C. et al. PD-1/PD-L1 blockade together with vaccine therapy facilitates effector T-cell infiltration into pancreatic tumors. J. Immunother. 38, 1-11 (2015).

142. Son, J. et al. Glutamine supports pancreatic cancer growth through a KRAS-regulated metabolic pathway. Nature 496, 101-105 (2013).

Acknowledgements

This work was supported by CA179991 (C.A.I.-D.) Melanoma Research Alliance \#305021 (C.A.I.-D.), F31 CA180682 (A.M.-M.) and T32 CA160001 (A.M.-M.).

Competing interests statement

The authors declare no competing interests. 